\title{
Relation between Phonological Processing, Auditory Processing and Speech Perception among Bilingual Poor Readers
}

\author{
Mohan Kumar Kalaiah \\ Department of Audiology and Speech Language Pathology, Kasturba Medical College (Manipal University), \\ Mangalore, Karnataka, India
}

\author{
Received June 8, 2015 \\ Revised August 18, 2015 \\ Accepted September 3, 2015
}

Address for correspondence

Mohan Kumar Kalaiah, MSc

Department of Audiology and Speech Language Pathology,

Kasturba Medical College

(Manipal University),

Mangalore 575 001, Karnataka, India

Tel +91-88840-45381

Fax +91-824-2428379

E-mail mohan.kumark@manipal. edu
Background and Objectives: A deficit in phonological processing abilities has been hypothesized as a cause of reading deficits among poor readers, but the precise etiology of this deficit is still unknown. Many studies have investigated the relation of auditory processing and speech perception with phonological processing, while the relation between these are not well understood. Thus, the present study was carried out to investigate the relation between these abilities among poor readers. Subjects and Methods: A total of 20 children between 7-12 years of age participated in the study. Among these 10 were typically developing children and 10 were poor readers. Auditory processing, speech perception in noise and phonological processing skills were assessed in both the groups. Results: Auditory processing was not significantly different between children in both the groups. In contrast, phonological awareness, verbal short-term memory and rapid automatized naming, which reflect phonological processing, and speech perception in noise were found to be significantly affected in poor readers. In addition, the results showed a significant correlation between phonological processing and speech perception in noise. Conclusions: The present study found a significant relationship between speech perception in noise and phonological processing, while there was no relationship between auditory processing and phonological processing. This finding suggests that poor speech perception among poor readers may be one of the contributing factors for phonological processing deficits, which in turn leads to reading difficulties.

J Audiol Otol 2015;19(3):125-131

KEY WORDS: Auditory processing · Phonological processing · Poor readers Reading impairment $\cdot$ Speech perception.

\section{Introduction}

Phonological processing skills are believed to play an important role in the development of reading [1-3], deficits in phonological processing forms a core deficit, leading to reading difficulties, among poor readers $[4,5]$. It consists of three main components namely: phonological awareness, phonological or verbal short-term memory, and rapid automatized

This is an Open Access article distributed under the terms of the Creative Commons Attribution Non-Commercial License (http://creativecommons. org/licenses/by-nc/3.0/) which permits unrestricted non-commercial use, distribution, and reproduction in any medium, provided the original work is properly cited. naming. Phonological awareness refers to the conscious ability to access and manipulate the individual phonemes of speech; phonological or verbal short-term memory codes auditory information for temporary storage; and rapid naming is the ability for efficient retrieval of phonological information from long-term memory. These skills are found to have stronger association with reading abilities, and differentially predicts reading ability. Deficits in phonological awareness, phonological memory, and rapid naming are common in children with reading impairments and these deficits are regarded as cause for reading difficulties faced by poor readers [6].

The phonological processing deficits among poor readers has been argued to arise from underlying auditory processing 
deficits [7-9], and various theories have been proposed to explain how auditory processing deficits can lead to phonological processing deficits [7-9]. Tallal [7] auditory temporal processing deficit hypothesis suggests that the reading impairment is a consequence of primary perceptual deficit that processes rapidly changing auditory information or rapid spectrotemporal characteristics of phonemes and sounds [8]. This perceptual deficit has been hypothesized to cause difficulty in analyzing speech at the phonemic level, resulting in poor phonemic segmentation and recoding skills leading to reading deficits in poor readers. In contrast, temporal sampling theory proposed by Goswami [9] suggests that phonological deficits found in poor readers may arise due to impaired auditory oscillatory phase-locking to slower temporal modulations below $10 \mathrm{~Hz}$ in auditory cortex. This, underlying auditory deficit has been hypothesized to affect the efficient recovery of syllabic structure from the speech signal, including supra-segmental and prosodic structure, resulting in reading difficulties. Thus, both auditory temporal processing deficit hypothesis and temporal sampling theory attributes auditory processing deficits to poor phonological processing which further leads to reading deficits.

Based on the individual data reported by various investigators, Ramus [5] noted that only $39 \%$ of the individuals with reading difficulties had auditory deficits. This finding suggests that only a subgroup of individuals with reading problems may have auditory processing deficits, on this basis auditory deficit based theories have been criticized [5]. Other than auditory deficit based theories, theories based on phonological deficit has been proposed to explain reading difficulties among poor readers. Phonological deficit hypothesis $[5,10]$ attributes reading deficits to a core deficit in phonological awareness. It proposes that individuals with reading deficits have specific impairments in the representation, storage and/ or retrieval of speech sounds. Further, it explains that the deficit in phonological awareness leads to difficulty learning grapheme-phoneme correspondence early on and to later difficulty learning decoding skills. But, proponents of auditory deficit based theories, on the other hand, have argued the phonological processing deficit as a symptom of an underlying auditory temporal processing deficit.

In addition to deficits in auditory and phonological processing, poor speech perception is also reported by various investigations among poor readers $[11,12]$. While, poor speech perception is found to be present only in a subset of poor readers $[5,13]$. Various studies have investigated the relationship between auditory processing and speech perception with phonological processing among poor readers. Results of these investigations have found a significant relationship between auditory processing, speech perception and phonological processing, but the direction of the relationship is not well understood [14]. Thus, in the present investigation we attempt to investigate the relationship between these abilities among poor readers.

\section{Subjects and Methods}

\section{Participants}

A total of 10 children identified to have reading difficulties and 10 typically developing children with no reports of reading, speech and language difficulties between 7-12 years of age participated in the study. Children with reading difficulties had language-based learning disability and were enrolled for intervention program, these children were referred as poor readers. The presence of language and reading impairment was established using Speech and Language Development Chart [15] or Assessment of Language Development test [16] and Early Reading Skills test respectively. All children had normal hearing in both ears with pure-tone thresholds less than $15 \mathrm{~dB}$ HL at octave frequencies from $250 \mathrm{~Hz}$ to $8000 \mathrm{~Hz}$ and speech identification scores greater than $90 \%$ at $40 \mathrm{~dB}$ SL (e.g., pure-tone average at 500, 1000, and $2000 \mathrm{~Hz}$ ) in quiet. None of the children had history of otologic disease. Typically developing children were matched with poor readers based on age, gender, educational environment, i.e., same class, and parental educational level. Ethical approval was obtained from institutional ethics committee of Kasturba Medical College, Mangalore to carry out the present study.

\section{Stimuli and procedure}

\section{Auditory processing}

Auditory processing of children was measured using frequency modulation detection and gap detection tasks. Frequency modulation detection was used to assess processing of slow varying dynamic stimuli and gap detection task was used to assess processing of rapid and brief signals. Stimuli used for assessing auditory processing were generated using Matlab 2009b (Mathworks Inc., Natick, MA, USA) at a sampling frequency of $44,100 \mathrm{~Hz}$ and 16-bit resolution. Both tasks were carried out using APEX 3 program (Leuven, Belgium) [17] installed on a personal computer, and the stimuli were presented monaurally at comfortable level using Sennheiser HD180 circum-aural headphones. Practice trials were given to each child to make them familiar with the task and also to obtain accurate thresholds. 
Frequency modulation

Frequency modulation detection threshold was obtained using a frequency modulated pure-tone, with carrier frequency of $1000 \mathrm{~Hz}$, sinusoidally modulated at $2 \mathrm{~Hz}$. The minimum depth of frequency change required to detect the modulation was defined as the frequency modulation detection threshold. The frequency modulation detection threshold of all the participants were obtained using three-interval, three alternative forced-choice procedure by means of two-down, one-up adaptive procedure. On each trial, one target and two standard stimuli were presented in a random sequence, and the participants were asked to identify the interval containing modulated tone among the three tones. The duration of all the stimuli were $500 \mathrm{msec}$ and were presented with an inter stimulus interval of $500 \mathrm{msec}$. Initially, the modulation depth was decreased by a factor of 1.2 from 100 to $11 \mathrm{~Hz}$ and a step size of $1 \mathrm{~Hz}$ was used from $11 \mathrm{~Hz}$ to $1 \mathrm{~Hz}$. Threshold run was terminated after eight reversals, and the mean of last six reversals in a block of eight was taken as threshold.

\section{Gap detection}

White noise of $500 \mathrm{msec}$ duration with and without gaps of variable duration were used to measure gap detection threshold of the participants. White noise with gap was obtained by introducing silence in the middle of white noise. The duration of gap was varied from $50 \mathrm{msec}$ to $1 \mathrm{msec}$ at a step size of 1 msec. All noise signals were windowed (20 msec hamming) at onset and offset and the duration of all the signals were 500 msec. Gap detection thresholds were obtained using three-interval, three-alternative forced-choice paradigm and twodown one-up adaptive staircase procedure. The noise signals were presented in a random order with an inter-stimulus interval of $500 \mathrm{msec}$, and participants task was to identify the interval containing noise with gap. Initially, the gap duration was $50 \mathrm{msec}$ and it was decreased by $5 \mathrm{msec}$, after the first reversal the step size was reduced to $1 \mathrm{msec}$. The mean of last six reversals in a block of eight was taken as gap detection threshold.

\section{Phonological processing}

\section{Phonological awareness}

Four subtests from phonological awareness test [18], rhyme recognition and production, syllable segmentation and blending, were used to measure phonological awareness. These tasks were administered and scored as recommended in the test material.
Verbal short-term memory

Verbal short-term memory was measured using non-word repetition task. Non-words were taken from Dynamic Indicators of Basic Early Literacy Skills [19]. The words were spoken by female talker and the utterances were digitally recorded using a sampling frequency of $44100 \mathrm{~Hz}$ and 16 bit resolution. Praat software (Amsterdam, the Netherlands) was used for recording and editing the utterances. A total of 25 non-words were presented individually and children were asked to repeat the words verbally. The number of words correctly repeated by children was noted and percentage correct score was computed.

\section{Rapid automatized naming}

Rapid digit naming task from Rapid automatized naming and Rapid alternating stimulus test [20] was used to measure rapid automatized naming. This task was administered and scored as recommended in the test material.

\section{Speech perception in noise}

Speech perception in noise was measured using English spondee words at a signal-to-noise ratio (SNR) of +5 and -5 $\mathrm{dB}$. The words were spoken by a young male in an acoustically treated room and were digitally recorded using sampling frequency of $44100 \mathrm{~Hz}$ and 16 bit resolution. These words were mixed with speech-spectrum-shaped noise by adjusting its root mean square (RMS) amplitude with reference to the RMS amplitude of noise to achieve the desired SNRs of +5 and $-5 \mathrm{~dB}$. The speech-spectrum-shaped noise was generated by randomizing the phase of the Fourier spectrum of concatenated words of original signals. Speech identification in noise task was used to measure speech perception in noise abilities of the participants. A total of 25 spondee words were presented binaurally along with speech-spectrumshaped noise at comfortable level using Sennheiser HD180 circum-aural headphones. Children were instructed to repeat the words, and the number of words correctly repeated was noted and percentage correct scores were computed for both SNRs. Prior to the data collection all children underwent training phase to make them familiar with the task. In training phase. 10 words were presented in noise and children were encouraged to repeat the words.

\section{Statistical analysis}

Descriptive statistics was carried out to obtain mean and standard deviation for all the measures. Prior to data analysis, the percent correct identification scores (speech perception in noise and non-word recognition) of each participant was converted into rationalized arcsine unit units. The data 


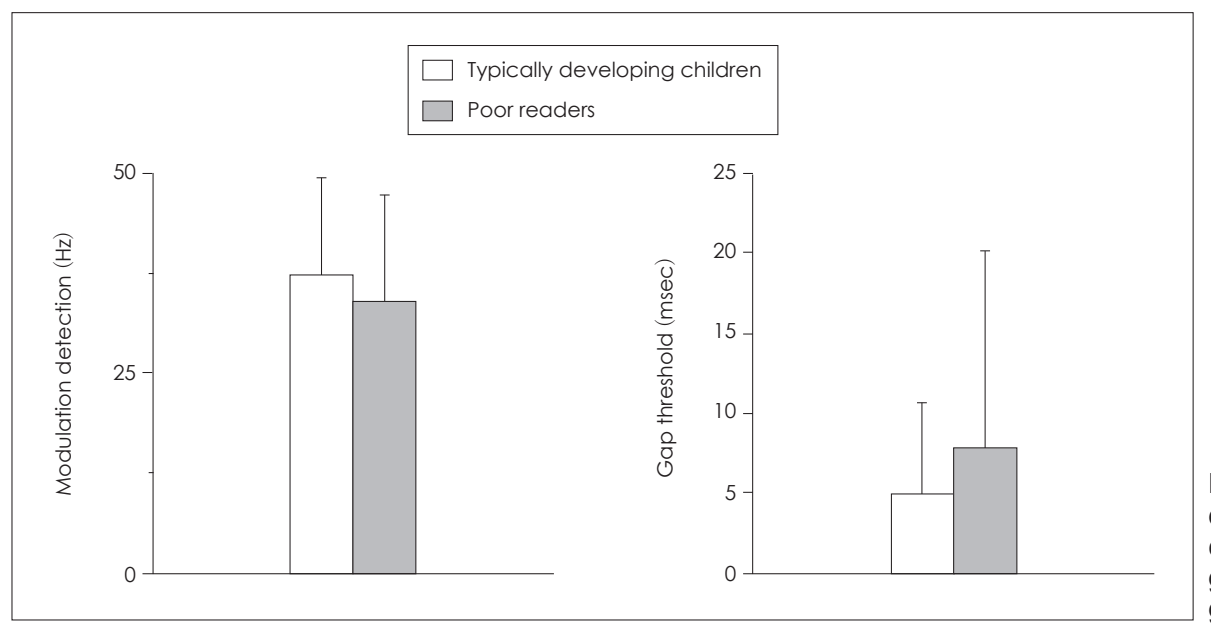

Fig. 1. Mean threshold and standard deviation (in parenthesis) for frequency modulation detection (left) and gap detection tasks (right) across the groups of participants. was subjected to Shapiro-Wilk test to check for normal distribution of data, result showed that the data was not normally distributed. Since the data was not normally distributed, Mann-Whitney's non-parametric test was carried out to investigate if the mean scores are significantly different between the groups. All statistical analysis was done using Statistical Package for the Social Sciences 16.0 (SPSS 16.0; SPSS Inc., Chicago, IL, USA).

\section{Results}

\section{Auditory processing}

Fig. 1 shows mean frequency modulation detection threshold and gap detection threshold of poor readers and typically developing children. In Fig. 1 it can be noted that the mean gap detection threshold is poorer among poor readers than typically developing children. In contrast, the mean frequency modulation detection threshold is slightly better among poor readers than typically developing children. To investigate if the mean thresholds are significantly different between the groups the data was subjected to Mann-Whitney U test. Results showed no significant difference between the groups $(p>0.05)$ for both gap detection and frequency modulation detection thresholds.

\section{Phonological processing abilities}

\section{Phonological awareness}

Fig. 2 shows the mean scores of poor readers and typically developing children for tasks measuring phonological awareness. From the figure it can be observed that the mean score of poor readers is poorer than typically developing children for rhyme recognition and rhyme production tasks. But, the mean score for segmentation is better among poor readers

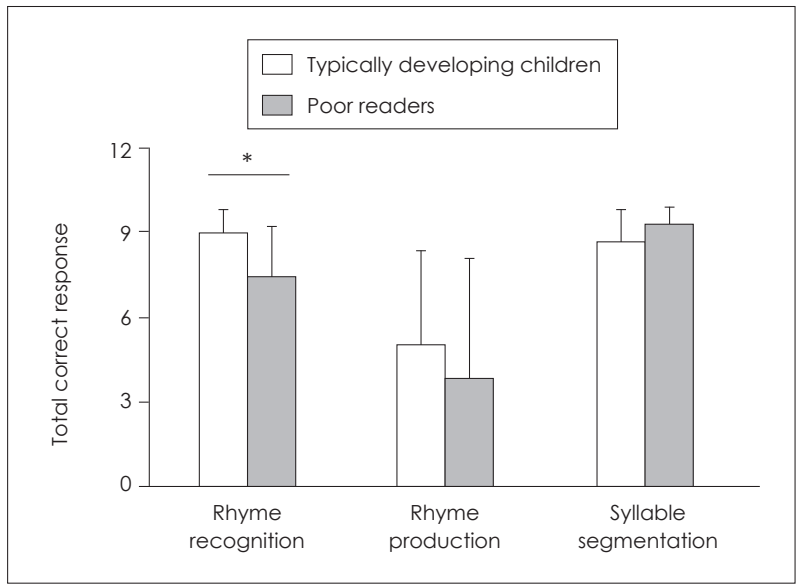

Fig. 2. Mean scores and standard deviation (in parenthesis) for various tasks measuring phonological awareness. ${ }^{*}$ significant difference between the groups at $p<0.05$.

than typically developing children. Mann-Whitney test revealed significant difference between the groups for rhyme recognition ( $U=21.5, p<0.05$ ), while the mean scores were not significantly different for rhyme production and segmentation $(p>0.05)$.

\section{Verbal short-term memory}

Fig. 3 shows the mean non-word recognition scores of children in two groups. In the figure it can be observed that the non-word recognition score is lower among poor readers compared to typically developing children. Mann-Whitney test showed no significant difference between the groups for nonword recognition scores.

\section{Rapid automatized naming}

Fig. 4 shows average duration required for poor readers and typically developing children to complete the tasks measuring rapid automatized naming. In the figure it is evident that the 


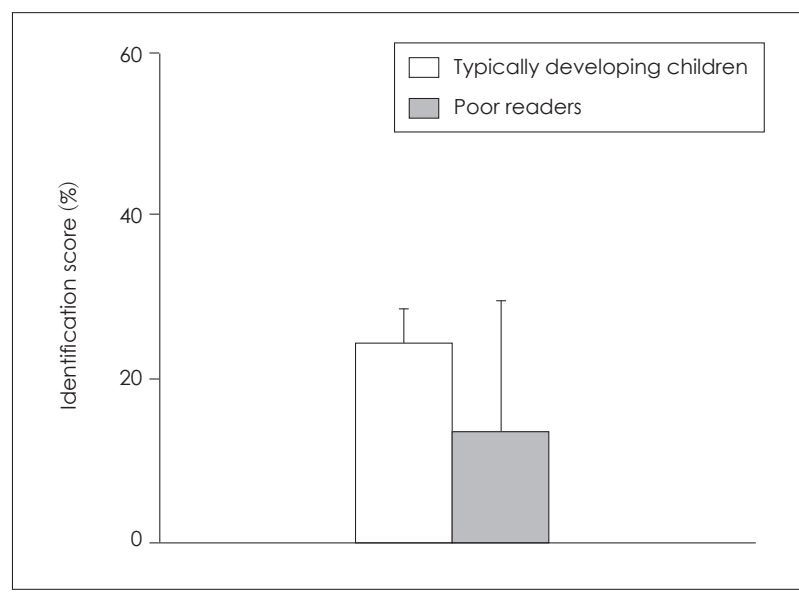

Fig. 3. Mean identification scores and standard deviation (in parenthesis) for non-word recognition task across groups.

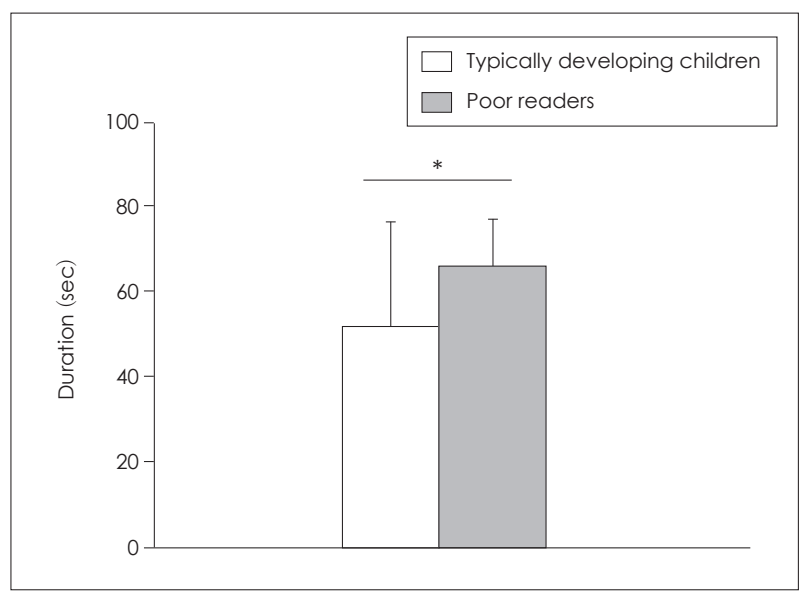

Fig. 4. Mean duration and standard deviation (in parenthesis) required for poor readers and typically developing children to complete the tasks measuring rapid automatized naming. *significant difference between the groups at $p<0.05$.

time to complete the task by poor readers is longer than typically developing children. Mann-Whitney test showed significant difference between the groups for rapid automatized naming $(\mathrm{U}=9, p<0.05)$.

\section{Speech perception in noise}

The mean speech identification score in noise for both groups are shown in Fig. 5. It shows that, the mean identification scores of poor readers is lower than typically developing children at both +5 and $-5 \mathrm{~dB}$ SNRs. Further, the result of Mann-Whitney test showed significant difference between the groups for mean speech identification scores at both +5 $\mathrm{dB}(\mathrm{U}=10, p<0.05)$ and $-5 \mathrm{~dB}(\mathrm{U}=14.5, p<0.05)$ SNRs.

\section{Relation between auditory processing, speech percep- tion, and phonological processing}

To investigate the relationship between auditory processing

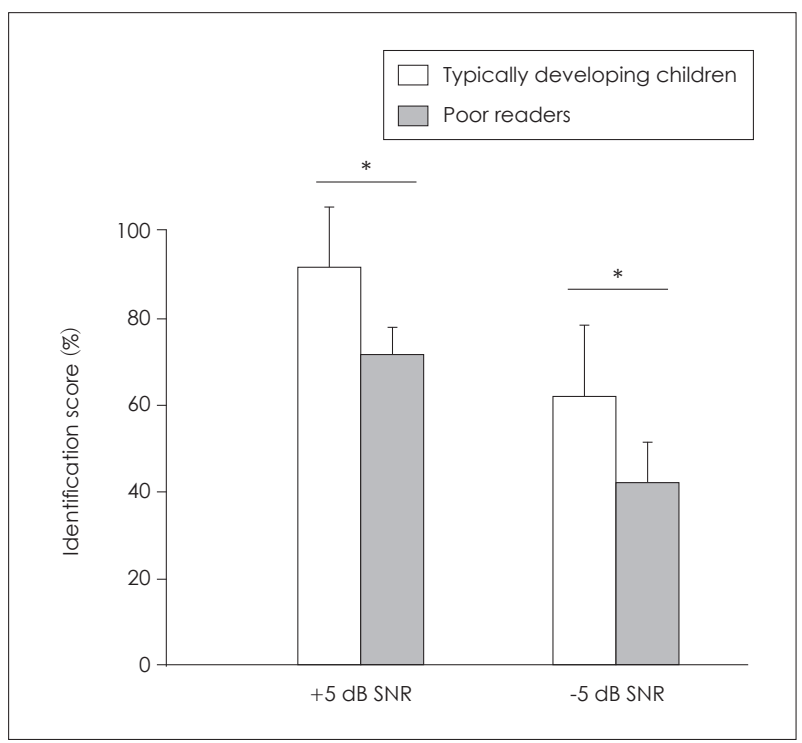

Fig. 5. Mean speech identification scores in noise and standard deviation (in parenthesis) across two groups of children. *significant difference between the groups at $p<0.05$. SNR: signal-to-noise ratio.

and speech perception in noise with phonological processing, spearmen product moment correlation analysis was carried out. Result showed a significant moderate negative correlation between speech perception in noise (at $-5 \mathrm{~dB}$ SNR) and rapid automatized naming $(\mathrm{r}=-0.519, p<0.05)$. In addition, a significant moderate positive correlation was present between verbal short-term memory and phonological awareness, i.e., with rhyme recognition $(\mathrm{r}=0.484, p<0.05)$ and rhyme production $(\mathrm{r}=0.499, p<0.05)$. Further, there was no significant correlation between auditory processing and phonological processing.

\section{Discussion}

The result of the present study showed no significant difference for auditory processing abilities between children with and without learning difficulties, and this finding is in consonance with the results of other investigations $[5,21,22]$. This finding suggests no relation between reading difficulties and auditory processing, similar suggestion has been made by other investigators $[5,22]$. However, in contrast to the findings in present study, a significant auditory processing deficit has been reported in children with learning difficulties using tasks such as auditory rise time detection [23] and frequency discrimination [24]. Thus, although the result of the present study shows no significant difference between the groups for auditory processing, the presence of auditory processing deficits cannot be ruled out since there is a possibility of other auditory processing tasks revealing the presence of auditory processing deficits.

The results further showed a significant phonological pro- 
cessing deficit and speech perception deficit to be present in poor readers. Similar deficits have been reported previously by other investigators for phonological processing $[25,26]$ and speech perception $[11,13,25,27]$ in children with reading difficulties. Thus, the above results obtained in the present study is the most consistent findings reported by various investigators across the studies in children with reading difficulties. Poor speech perception among poor readers may be attributed to 1) difficulties perceiving the stimuli, or 2) difficulties producing them quickly and accurately, or 3) difficulties in encoding the stimuli that may be common to all phonological tasks [28]. Recently, Ziegler, et al. [11] showed normal masking release in children with dyslexia, who had significantly poorer speech perception in noise. This findings shows lack of peripheral deficits which could affect speech perception in noise among children with dyslexia. Thus, authors attributed poor speech perception to lack of speech robustness in noisy listening conditions [11]. Correlation analysis in the present study showed a significant negative correlation between speech perception in noise and phonological processing ability. Similar finding has been reported by various investigators among poor readers suggesting that development of phonological processing is dependent on speech perception $[13,29]$. Thus, based on the findings of the present investigation we suggest that normal speech perception ability may be important for normal development of phonological processing, which is critical for development of reading ability.

The present study shows that speech perception may be important for normal development of phonological processing. Based on this finding it may be interesting to investigate whether the use of signal enhancing strategies, which improve SNR in noisy listening situations, positively improve reading skills among poor readers. McSporran, et al. [30] investigated the benefit of sound field amplification on listening behaviour of children who had failure in academic performance. The study showed a significant increase in listening behaviour of children following use of sound field amplification for five months. Similar benefit has been reported for development of literacy skills by various investigators for children with hearing loss [31], aboriginal students [32], and typically developing children [33,34]. Purdy, et al. [35] evaluated the benefit of personal FM system for children with reading delay, no significant improvement was observed in reading age of children after using the FM system for six months. The authors attributed the lack of improvement to use of FM systems for shorter duration and lack of comprehensive in-depth reading assessment. Though there was no significant improvement, the study found listening benefits for children with reading delay thus it was speculated that FM systems may be ben- eficial for children with reading delay to improve reading abilities. In another study, Hornickel, et al. [36] found classroom FM system to improve reading and phonological awareness abilities in children with dyslexia. From these above studies it is evident that sound field amplification systems and personal FM systems are beneficial to improve literacy skills of children in classrooms. However, there are limited studies investigating the benefits of these devices for poor readers, hence, further research is required to understand if sound field amplification system and personal FM systems are beneficial for poor readers in classrooms.

To conclude, the present study found a significant relation between speech perception in noise and phonological processing, while there was no relation between auditory processing and phonological processing. In addition, the study found no difference in auditory processing ability between typically developing children and poor readers. This finding suggests that poor speech perception ability among poor readers may be one of the contributing factors for phonological processing deficits, which results in reading difficulties. Assistive listening devices (such as sound field amplification system, personal FM system) which improves SNR are shown to improve listening behaviors and reading abilities among poor readers, while conclusive evidence is not present. Hence, further research is required to understand the effects of assistive listening devices on learning among poor readers.

\section{Acknowledgments}

Thanks to Dean, Kasturba Medical College, Mangalore (Manipal University), and Head of Department, Department of Audiology and Speech Language Pathology, Kasturba Medical College, Mangalore (Manipal University) for permitting us to conduct the study.

\section{REFERENCES}

1) Wagner RK, Torgesen JK, Laughon P, Simmons K, Rashotte CA. Development of young readers' phonological processing abilities. J Educ Psychol 1993;85:83-103.

2) Sprugevica I, Høien T. Relations between enabling skills and reading comprehension: a follow-up study of Latvian students from first to second grade. Scand J Psychol 2004;45:115-22.

3) Nelson JM, Lindstrom JH, Lindstrom W, Denis D. The Structure of Phonological Processing and Its Relationship to Basic Reading. Exceptionality 2012;20:179-96.

4) Snowling MJ. Dyslexia. 2nd ed. Oxford: Wiley;2000.

5) Ramus F. Developmental dyslexia: specific phonological deficit or general sensorimotor dysfunction? Curr Opin Neurobiol 2003;13: 212-8.

6) Stanovich KE, Siegel LS. Phenotypic performance profile of children with reading disabilities: a regression-based test of the phonological-core variable-difference model. J Educ Psychol 1994;86:2453.

7) Tallal P. Improving language and literacy is a matter of time. Nat Rev Neurosci 2004;5:721-8.

8) Tallal P, Gaab N. Dynamic auditory processing, musical experience and language development. Trends Neurosci 2006;29:382-90. 
9) Goswami U. A temporal sampling framework for developmental dyslexia. Trends Cogn Sci 2011;15:3-10.

10) Snowling MJ. From language to reading and dyslexia. Dyslexia 2001;7:37-46.

11) Ziegler JC, Pech-Georgel C, George F, Lorenzi C. Speech-perception-in-noise deficits in dyslexia. Dev Sci 2009;12:732-45.

12) Bradlow AR, Kraus N, Hayes E. Speaking clearly for children with learning disabilities: sentence perception in noise. J Speech Lang Hear Res 2003;46:80-97.

13) Manis FR, Mcbride-Chang C, Seidenberg MS, Keating P, Doi LM, Munson B, et al. Are speech perception deficits associated with developmental dyslexia? J Exp Child Psychol 1997;66:211-35.

14) Boets B, Vandermosten M, Poelmans H, Luts H, Wouters J, Ghesquière P. Preschool impairments in auditory processing and speech perception uniquely predict future reading problems. Res Dev Disabil 2011;32:560-70.

15) Gard A, Gorman J, Gilman L. Speech and language development chart. Austin, TX: PRO-ED;1993.

16) Lakkanna S, Venkatesh K, Bhat JS. Assessment of Language Development. Bakersfield, CA: Omni Therapy Services;2008.

17) Francart T, van Wieringen A, Wouters J. APEX 3: a multi-purpose test platform for auditory psychophysical experiments. J Neurosci Methods 2008;172:283-93.

18) Robertson C, Salter W. The phonological awareness test. East Moline, IL: LinguiSystems;1997.

19) Good RH, Kaminski RA, Smith S, Laimon D. Dynamic Indicators of Basic Early Literacy Skills (DIBELS). 5th ed. Eugene, OR: Institute for the Development of Educational Achievement, University of Oregon;2001.

20) Wolf M, Denckla MB. RAN/RAS: Rapid Automatized Naming and Rapid Alternating Stimulus Tests. Austin, TX: PRO-ED;2005.

21) Vandewalle E, Boets B, Ghesquière P, Zink I. Auditory processing and speech perception in children with specific language impairment: relations with oral language and literacy skills. Res Dev Disabil 2012; 33:635-44.

22) Vellutino FR, Fletcher JM, Snowling MJ, Scanlon DM. Specific reading disability (dyslexia): what have we learned in the past four decades? J Child Psychol Psychiatry 2004;45:2-40.

23) Fraser J, Goswami U, Conti-Ramsden G. Dyslexia and Specific
Language Impairment: The Role of Phonology and Auditory Processing. Sci Stud Read 2010;14:8-29.

24) McArthur GM, Bishop DV. Frequency discrimination deficits in people with specific language impairment: reliability, validity, and linguistic correlates. J Speech Lang Hear Res 2004;47:527-41.

25) Snowling MJ, Stackhouse J, Rack J. Phonological dyslexia and dysgraphia - a developmental analysis. Cogn Neuropsychol 1986;3; 309-39.

26) Melby-Lervåg M, Lyster SA, Hulme C. Phonological skills and their role in learning to read: a meta-analytic review. Psychol Bull 2012; 138:322-52.

27) Brady S, Shankweiler D, Mann V. Speech perception and memory coding in relation to reading ability. J Exp Child Psychol 1983;35: 345-67.

28) Brady S, Poggie E, Rapala MM. Speech repetition abilities in children who differ in reading skill. Lang Speech 1989;32(Pt 2):109-22.

29) Hurford DP. Training phonemic segmentation ability with a phonemic discrimination intervention in second- and third-grade children with reading disabilities. J Learn Disabil 1990;23:564-9.

30) McSporran E, Butterworth Y, Rowson VJ. Sound Field Amplification and Listening Behaviour in the Classroom. Br Educ Res J 1997; 23:81-96.

31) Jones J, Berg F, Viehweg S. Listening of kindergarten students under close, distant, and sound field FM amplification conditions. Educ Audiol Monogr 1989;1:56-65.

32) Flexer C. The startling possibility of soundfield. Adv Speech-Language Pathol Audiol 2000;10:13.

33) Heeney MF. Classroom sound field amplification, listening and learning. Australia: University of Newcastle; 2007.

34) Rosenberg GG, Blake-Rahter P, Heavner J, Allen L, Redmond BM, Phillips J, et al. Improving Classroom Acoustics (ICA): A ThreeYear FM Sound Field Classroom Amplification Study. J Educ Audiol 1999;7:8-28.

35) Purdy SC, Smart JL, Baily M, Sharma M. Do children with reading delay benefit from the use of personal FM systems in the classroom? Int J Audiol 2009;48:843-52.

36) Hornickel J, Zecker SG, Bradlow AR, Kraus N. Assistive listening devices drive neuroplasticity in children with dyslexia. Proc Natl Acad Sci U S A 2012;109:16731-6. 